\title{
Fiber optic distributed temperature sensor mapping of a jet-mixing flow field
}

\author{
S. Lomperski $\cdot$ C. Gerardi $\cdot$ W. D. Pointer
}

Received: 19 August 2014 / Revised: 6 February 2015 / Accepted: 6 February 2015 / Published online: 4 March 2015

(c) The Author(s) 2015. This article is published with open access at Springerlink.com

\begin{abstract}
This paper introduces the use of a Rayleigh backscatter-based distributed fiber optic sensor to map the temperature field in air flow for a thermal fatigue application. The experiment involves a pair of air jets at 22 and $70{ }^{\circ} \mathrm{C}$ discharging from $136 \mathrm{~mm}$ hexagonal channels into a $1 \times 1 \times 1.7 \mathrm{~m}$ tank at atmospheric pressure. A $40 \mathrm{~m}$-long, $\phi 155 \mu \mathrm{m}$ fiber optic sensor was wound back and forth across the tank midplane to form 16 horizontal measurement sections with a vertical spacing of $51 \mathrm{~mm}$. This configuration generated a 2D temperature map with 2800 data points over a $0.76 \times 1.7 \mathrm{~m}$ plane. Fiber optic sensor readings were combined with PIV and infrared measurements to relate flow field characteristics to the thermal signature of the tank lid. The paper includes sensor stability data and notes issues encountered using the distributed temperature sensor in a flow field. Sensors are sensitive to strain and humidity, and so accuracy relies upon strict control of both.
\end{abstract}

\section{Introduction}

A typical electric power station uses high-temperature fluids to turn turbines that generate electricity. Routine operations like startup and maintenance shutdowns thermally

S. Lomperski $(\bowtie) \cdot$ C. Gerardi

Argonne National Laboratory, 9700 S. Cass Ave.,

Argonne, IL, USA

e-mail: lomperski@anl.gov

C. Gerardi

e-mail: cgerardi@anl.gov

W. D. Pointer

Oak Ridge National Laboratory, P.O. Box 2008,

Oak Ridge, TN, USA

e-mail: pointerwd@ornl.gov cycle many of the high-pressure piping components. Piping and pressure vessels are usually fabricated from steel, and thermal cycling of structural steels can generate progressive material degradation that may lead to component failure. Routine thermal transients are part of a plant's design basis, but unanticipated cycling can cause unexpected failures. For example, unforeseen temperature fluctuations in components are used to mix hot and cold fluid streams. Thermal fatigue failures in power plants have been observed in boiler tubes (Ahmad et al. 2010), a steam exhaust silencer (Lee 1995) and mixing tees of nuclear power stations (Blondet and Faidy 2002; Yetisir 2009). In addition, thermal shock and failure of high-pressure components can result from unexpected thermal transients (Salonen et al. 2007). Even modest thermal cycling can weaken materials over the decades-long life of a power plant, and so reactor designers seek to minimize cycling to extend component service life.

Thermal fatigue is studied experimentally to guide strategies for limiting component damage of pressurized water reactors (e.g., Fissolo et al. 2009). In addition, computational fluid dynamics (CFD) tools are being used to simulate fluid/structure interactions to improve understanding and management of thermal cycling in nuclear power system components (Galpin and Simoneau 2011; Hannink and Blom 2011). Broader acceptance of these tools for plant design and licensing will require additional validation against experimental data, especially for cases involving complex interactions between interdependent physical phenomena. Rigorous validation of CFD tools requires data of high spatial and temporal resolution. Ideally, a large region of the flow field is mapped at high resolution to capture both local turbulence quantities and large-scale flow structures. The former supports physics modeling while the latter reveals flow phenomena at scales that concern 
engineers. Our experiments generate validation data to support development of turbulence models that will improve code efficiency and ability to predict the macroscopic phenomena of concern to plant designers. This paper describes implementation of a fiber optic distributed temperature sensor to map flow field temperature in these experiments with a resolution unattainable from conventional sensors such as thermocouples.

A typical CFD validation data set consists chiefly of temperature and velocity measurements across a precisely characterized geometry along with a set of boundary conditions. Velocity data can be obtained with particle image velocimetry (PIV), a familiar optical technique that is well-suited for CFD validation since it can generate high-resolution data without disturbing the flow. In contrast, it has proven difficult to map temperature fields with the comprehensiveness of PIV. One well-established optical temperature measurement technique involves laser-induced fluorescence (LIF). Like PIV, LIF employs lasers and cameras to make measurements across a plane illuminated by a light sheet. The laser excites tracers that fluoresce with a temperaturedependent intensity, emission spectrum, or decay time. Gas flows can be seeded with gases such as acetone (Bryant et al. 2000) or toluene (Cundy et al. 2011). For liquids, dissolved fluorescent dyes have been employed in temperature mapping of single phase liquids (Dahikar et al. 2010) and the two-phase field of a solidification process (Shafii et al. 2010). Simultaneous PIV and LIF can be accomplished with thermochromic liquid crystal tracers (Dabiri 2009) and luminescent particles for liquid flow (Someya et al. 2009) and gas flow (Abram et al. 2013). These are powerful measurement methods, but chemicals must be added to flows and calibration is more elaborate than for PIV. As a consequence, LIF is not as widely used as PIV and temperature fields are often mapped with far less detail than velocity fields even when comparable resolution would considerably increase the value of experiment data.

Other spectroscopic techniques can map temperature in air flows without special chemicals or seeding. Rayleigh scattering methods have been used for 2D mapping of flames (Zetterberg et al. 2008) and a natural circulation flow field between plates (Boguszko and Elliott 2005). Coherent anti-Stokes Raman spectroscopy (CARS) is widely used for gas phase temperature measurements in the combustion field and is capable of both 2D (Bohlin and Kliewer 2014) and 1D (Kulatilaka et al. 2011) mapping, though it is more commonly used for point measurements since three overlapping laser light fields are needed for the measurement volume. Tuneable diode laser absorption spectroscopy (TDLAS) measures narrow line absorption of gas species such as water vapor along the path of a laser beam (Cai et al. 2009; Allen 1998), providing an average temperature over the beam path. And 1D profiles can be obtained through wavelength multiplexing across multiple absorption bands (Liu et al. 2007). Rayleigh scattering and CARS techniques require relatively high-power lasers, while TDLAS can function with lowpower laser diodes.

Infrared thermography is another optical temperature measurement technique with temporal and spatial resolution suitable for CFD validation. Volino and Smith (1999) characterized free convection in a water tank with IR measurements of surface temperature and PIV of the subsurface velocity field. Sou et al. (2011) used IR and PIV to map coherent structures generated by an ultrasonic horn in a water tank. Neto et al. (2006) and Cehlin et al. (2002) mapped air temperature indirectly by placing a thin screen within the flow to measure its thermal signature. Gordge and Page (1993) made direct infrared measurements of air jet temperatures by adding $\mathrm{CO}_{2}$, while Narayanan et al. (2003) used $\mathrm{SF}_{6}$.

An alternative is available in distributed temperature sensing based on Rayleigh scattering and swept-wavelength interferometry, a relatively new technique and an offshoot of distributed strain sensing. Thousands of temperature measurements can be acquired along a single thin optical fiber. A distributed temperature sensor (DTS) can span large flow fields and function in environments that are unsuitable for image-based techniques. They can be wrapped around complex surfaces for 3D mapping that would be difficult to achieve with strictly planar measurements. This paper presents implementation of a Rayleigh scattering-type DTS in a thermal mixing experiment. It describes the measurement principle, setup and data for the jet-mixing configuration and sensor stability data. We also note implementation issues associated with using DTSs for flow field temperature mapping. The paper illustrates the ability of these sensors to complement PIV and enhance the value of data sets for CFD validation. We begin by describing the physical principles underlying the sensors used in this study.

\section{Sensing with scattered light}

Scattering mechanisms inevitably attenuate light waves as they travel along a fiber optic cable. Physical changes in the cable modulate the scattered light, which can be collected and analyzed to monitor the state of the cable along its entire length. A fiber optic cable may therefore serve as a "distributed" sensor. A variety of such sensors have been developed on the basis of Raman, Brillouin and Rayleigh scattering. Rayleigh scattering is generated by microscopic inhomogeneities in density and composition of the fiber 
core. Scattering is elastic, with no change in wavelength, and as a major source of attenuation in telecom fibers, the Rayleigh signal is relatively strong. Brillouin scattering arises from acoustic oscillations in waveguide density, while Raman scattering originates from light interactions with molecular vibrations. Both Brillouin and Raman scattering are inelastic, with so-called Stokes and anti-Stokes components corresponding to shifts away from the source wavelength. In all cases, sensing is accomplished by detecting variations in the scattering spectrum that accompany physical changes in the cable such as strain or temperature change.

Brillouin scattering-type sensors respond to both strain and temperature, while the Raman versions respond only to temperature. Both can be many kilometers long and have found use in measuring temperature within oil wells (Williams et al. 2000) and along power cables serving offshore wind farms (Fromme et al. 2011), as well as strain within infrastructure such as tunnels and pipelines (Rajeev et al. 2013). Brillouin systems can achieve spatial resolution down to $\sim 1 \mathrm{~cm}$, while Raman is limited to $\sim 1 \mathrm{~m}$; time response is generally on the order of minutes (Bao and Chen 2012) though some commercial systems can now achieve data rates of approximately $1 \mathrm{~Hz}$. Comprehensive reviews of the physics of fiber optic sensors can be found in (Bao and Chen 2012; Palmieri and Schenato 2013).

While enormous amounts of data can be generated by Raman and Brillouin scattering-based systems, spatial resolution and bandwidth are insufficient for most fluid dynamics experiments. Higher resolution can be realized instead with Rayleigh scattering-based systems, which can currently achieve data rates of hundreds of Hertz and spatial resolution to $\sim 1 \mathrm{~mm}$. Sensors are tens of meters long rather than kilometers, and so sensor length is traded for improved time and spatial resolution, both of which are highly valued in CFD validation experiments. The temperature sensors used in the present study exploit the Rayleigh scattering losses present in ordinary commercial telecom optical fibers.

\subsection{Swept-wavelength interferometry}

Light traveling through a fiber optic waveguide is scattered by impurities and structural variations at the molecular level. The random distribution of these inhomogeneities is stable, giving rise to a backscatter pattern that is unique to the fiber. The spectrum and amplitude of the pattern can be read to serve as a fiber signature and utilized to monitor the physical state of the cable.

In a technique known as swept-wavelength interferometry (SWI), a low-power tunable laser launches a narrow band signal into the fiber for the purpose of registering resultant backscatter (Gifford et al. 2007). Figure 1 illustrates the measurement principle. The laser, operating in the near-infrared around $1500 \mathrm{~nm}$, is swept across an interval of several nanometers. An optical coupler splits the signal between a reference leg and a measurement leg terminated with a sensor. Backscattered light from the sensor is combined with the reference leg signal and then split to polarization diverse detectors $\mathrm{S}$ and $\mathrm{P}$ to register the interference signals. Detector signals are digitized and summed to retrieve the Rayleigh scattering signal. The polarization controller and signal split into orthogonal polarization states ensure that the Rayleigh scattering signal is independent of the polarization state of the light scattered from the sensing fiber (Froggatt et al. 2006; Gifford et al. 2007). Figure 2 shows a sample scattering signal generated by one of the $40 \mathrm{~m}$-long sensors used for this study. A reflection is evident at the sensor input connector, while the end is marked by a sharp drop in signal strength from an end termination that diffuses the residual laser pulse to prevent its return to the detectors.

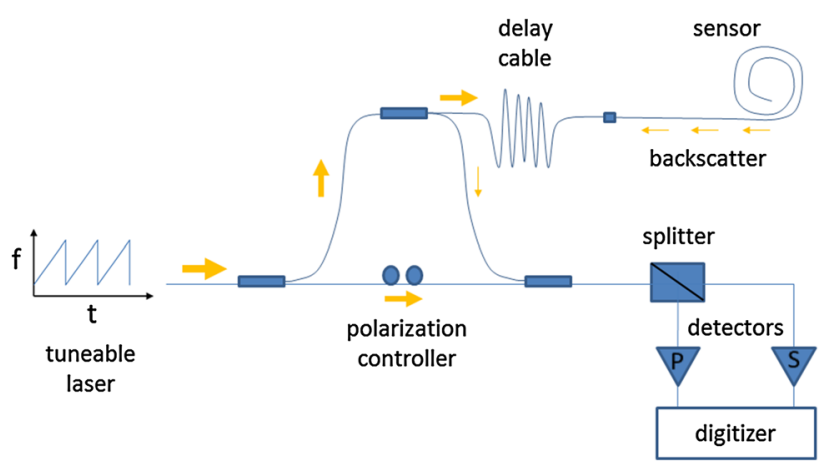

Fig. 1 Principle components of a swept-wavelength interferometer for measuring Rayleigh backscatter

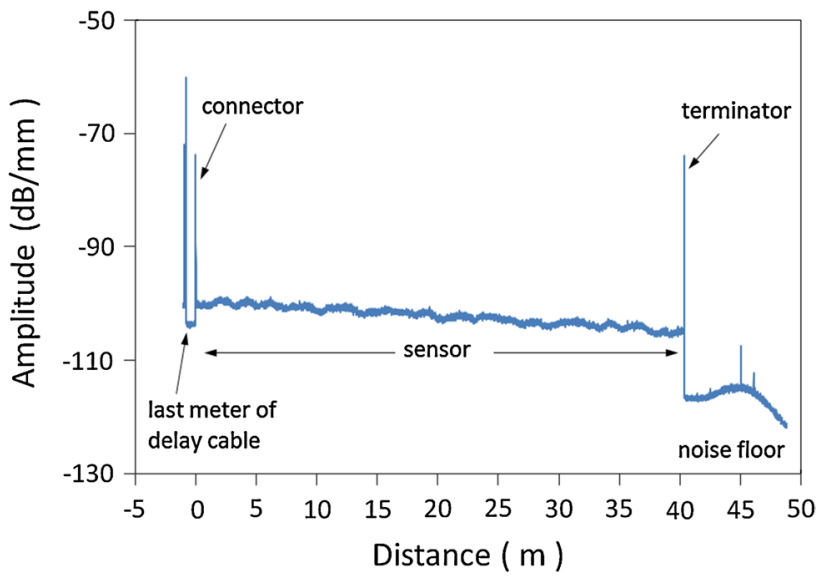

Fig. 2 Rayleigh scattering amplitude versus distance for a DTS used in this study. Sensor length $40 \mathrm{~m}$ 
The basic elements of SWI can be understood by considering the simple case of scattering from a single sensor scattering center. The electric field amplitude of the tuneable laser diode source can be written as:

$E(t)=E_{0} e^{-j \omega(t) t}$

The source signal is split into reference $E_{\mathrm{r}}(t)$ and scattering $E_{\mathrm{s}}(t)$ waveforms having delay $\tau$ and phase $\phi$ upon reaching the detector:

$$
\begin{aligned}
& E_{r}(t)=E_{r} e^{-j\left(\omega\left(t+\tau_{\mathrm{r}}\right) t+\phi_{\mathrm{r}}\right)} \\
& E_{\mathrm{S}}(t)=E_{\mathrm{S}} e^{-j\left(\omega\left(t+\tau_{\mathrm{s}}\right) t+\phi_{\mathrm{s}}\right)}
\end{aligned}
$$

Figure 3 depicts the signals at the detector, one from the reference leg and the other returning from an arbitrary sensor scattering center. The laser diode sweeps linearly between lower and upper wavelength limits, 1538-1542 nm for the $\pm 150{ }^{\circ} \mathrm{C}$ measurement range used in this study. The color band in the plot is intended to emphasize that optical frequency is being modulated rather than amplitude. The top curves illustrate how a difference in path length produces a constant delay between the two waveforms, resulting in a frequency difference at the detector (middle plot).

While the interference signal is the sum of the reference and scattering signals, photodetectors are square-law devices and output is proportional to the square of the electric field at the input:

$$
\begin{aligned}
I_{\mathrm{d}}(t) & \sim \operatorname{Re}\left[E_{\mathrm{r}}(t)+E_{\mathrm{S}}(t)\right]^{2} \\
I_{\mathrm{d}}(t) & \sim\left[E_{\mathrm{r}} \cos \left(\omega\left(t+\tau_{\mathrm{r}}\right)+\phi_{\mathrm{r}}\right)+E_{\mathrm{s}} \cos \left(\omega\left(t+\tau_{\mathrm{s}}\right)+\phi_{\mathrm{s}}\right)\right]^{2}
\end{aligned}
$$
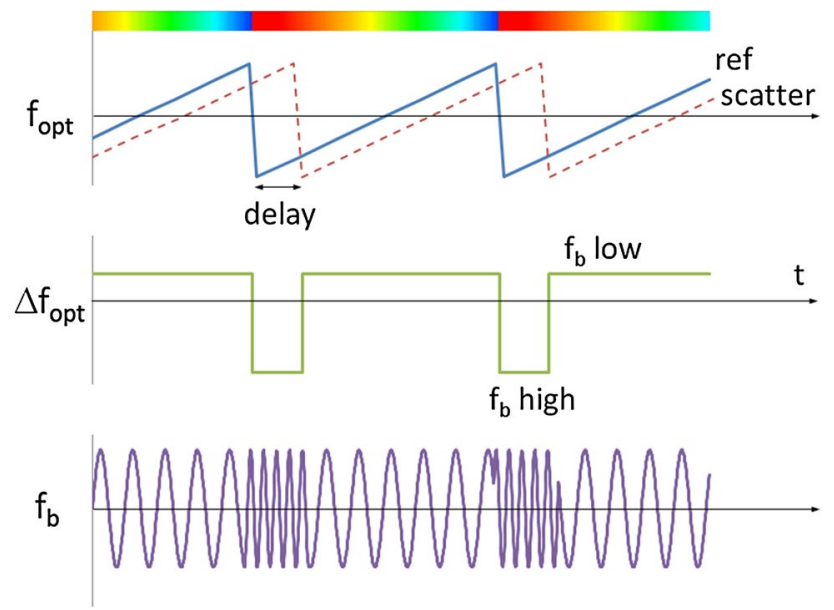

Fig. 3 Correspondence between interference spectrum at detector with optical signals originating from reference arm and single sensor scattering center. Top optical frequency modulation of reference and scattering signals; middle instantaneous difference in optical frequency; bottom interference waveform at detector. Adapted from Uttam and Culshaw (1985)
Writing $\omega\left(t+\tau_{\mathrm{r}}\right)$ as $\omega_{\mathrm{r}}$ and $\omega\left(t+\tau_{\mathrm{s}}\right)$ as $\omega_{\mathrm{s}}$, the interference signal is seen as a combination of low- and high-frequency components:

$$
\begin{aligned}
I_{\mathrm{d}}(t) \sim & E_{\mathrm{r}}^{2}+E_{\mathrm{S}}^{2}+E_{\mathrm{r}}^{2} \cos 2\left(\omega_{\mathrm{r}} t+\phi_{\mathrm{r}}\right)+E_{\mathrm{S}}^{2} \cos 2\left(\omega_{\mathrm{S}} t+\phi_{\mathrm{S}}\right) \\
& +2 E_{\mathrm{r}} E_{\mathrm{S}}\left[\cos \left(\left(\omega_{\mathrm{r}}+\omega_{\mathrm{S}}\right) t+\phi_{\mathrm{r}}\right)+\cos \left(\left(\omega_{\mathrm{r}}-\omega_{\mathrm{S}}\right) t+\phi_{\mathrm{S}}\right)\right]
\end{aligned}
$$

The first two terms are DC, while the following two are high frequency and beyond detector bandwidth. The final two terms contain elements with beat frequencies $\omega_{\mathrm{r}}+\omega_{\mathrm{s}}$ and $\omega_{\mathrm{r}}-\omega_{\mathrm{s} .}$ The former is again beyond detector bandwidth, but the latter is an intermediate frequency that carries the desired scattering information. This waveform beat frequency, illustrated at the bottom of Fig. 3, is used to identify scattering center position. This differs from timedomain reflectometry in which location is determined using time-of-flight measurements and signal propagation speed. The lower beat frequency is proportional to the scattering center distance $\mathrm{L}$ and fiber refractive index $n$ :

$f_{\mathrm{b}}=2 \operatorname{Ln} \gamma / c$

where $c$ is the speed of light and $\gamma$ is the laser diode frequency sweep rate in GHz/s. The highest beat frequencies correspond to scattering centers furthest from the sensor input.

The simple interference waveform in Fig. 3 represents the product of a single scattering center. An actual sensor with its continuum of scattering centers generates a far more complex signal. Scattering center location is mapped from beat frequencies using a fast Fourier transform of the interference signal (Froggatt and Moore 1998 and Gifford et al. 2007). A collection of scattering centers along a segment $\Delta \mathrm{L}$ is selected through a narrow frequency window $\Delta f_{\mathrm{b}}$. Spatial resolution is controlled via window width. The Rayleigh scattering spectrum of each segment is then analyzed to obtain strain or temperature, and the collection of sequential segments along the fiber optic cable constitutes the distributed sensor. The next section describes the basic principles involved in obtaining strain and temperature from a segment's scattering signal.

\subsection{Temperature sensing}

An optical fiber scattering spectrum such as that of Fig. 2 is random at the molecular level, essentially stable, and serves as a signature of the fiber. A fiber can serve as a sensor because changes in physical dimensions or refractive index shift the Rayleigh scattering spectrum in a repeatable manner. Strain and temperature changes generate such shifts, and so optical fibers can serve as strain or temperature sensors. The physical basis for the spectrum shift can be understood as that of a continuous, weak fiber Bragg 


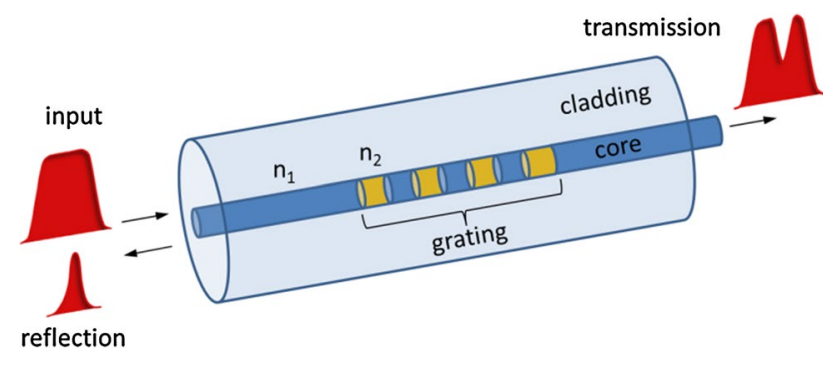

Fig. 4 Structure and operating principle of a fiber Bragg grating; reflection spectrum shifts as grating periodicity and/or indices of refraction change with strain or $\Delta T$

grating (Kreger et al. 2006). A fiber Bragg grating consists of periodic variations in the core refractive index along a section of the fiber optic waveguide (Bhatia 2001). Figure 4 shows the basic principle in which a grating reflects a fraction of the incident light back toward the source. Reflection spectrums are tailored through selection of grating periodicity and refractive indices. Straining a grating changes its periodicity and alters the reflected spectrum. Temperature variations affect the spectrum through changes in both periodicity and refractive indices (Bhatia 2001).

An analogous process occurs in a distributed fiber optic sensor as strain or temperature changes alter the relative positions of scattering centers along the fiber optic cable. Therefore, sensor response can be described by that of a fiber Bragg grating (Gifford et al. 2007; Yúkssel et al. 2011):

$\frac{\Delta \lambda}{\lambda}=K_{\mathrm{T}} \Delta T+K_{\mathrm{s}} \varepsilon$

where $K_{\mathrm{T}}$ and $K_{\varepsilon}$ are the temperature and strain coefficients, respectively. $K_{\mathrm{T}}$ includes coefficients for thermal expansion and the index of refraction. We are interested here in the temperature coefficient, which varies with material composition and is on the order of $8 \times 10^{-6} \mathrm{~K}^{-1}$ for silica optical fibers. This coefficient must be both stable and invariable along the length of the optical fiber if it is to be useful as a sensor.

Figure 5 shows a sample spectrum for a $10 \mathrm{~mm}$ segment of the sensor used for Fig. 2. Scattering amplitude versus optical wavelength is plotted for two cases: the first is a reference state at room temperature and the second with the sensor warmed by a heat gun. The plot shows the heatedstate spectrum to be an echo of the reference spectrum, shifted in wavelength with similar, though not identical amplitude features. The plot demonstrates the stable nature of the signature as it shifts in wavelength with $\Delta T$. Temperature range depends upon the width of the optical wavelength window and increases with window width. The window shown in the plot corresponds to a range of $\pm 150{ }^{\circ} \mathrm{C}$.

Quantitative measurement of temperature (or strain) is accomplished by cross-correlating the two spectrums to

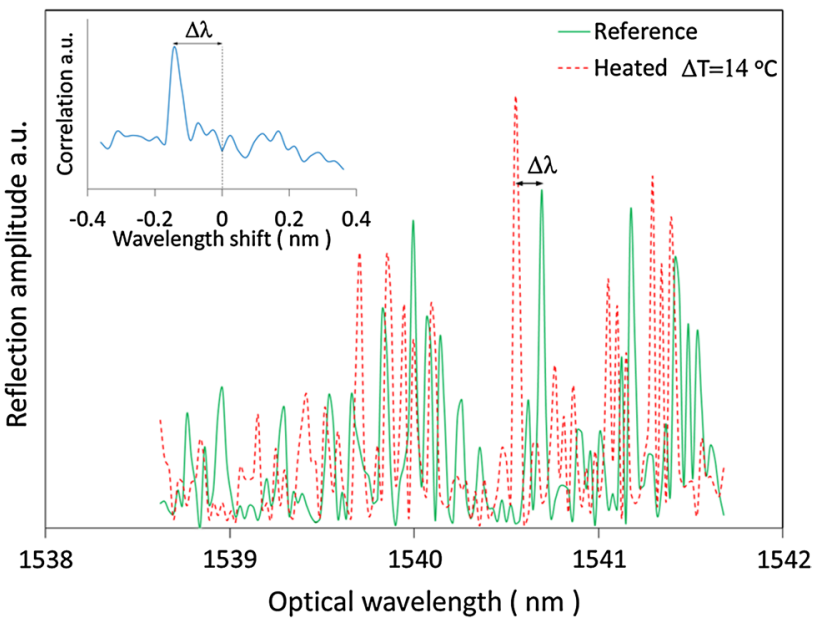

Fig. 5 Backscattered spectrum versus laser wavelength for a selected $10 \mathrm{~mm}$-long DTS segment. Inset cross-correlation of reference and heated spectrums

determine the magnitude of the wavelength shift. The plot inset in Fig. 5 shows the cross-correlation of the two spectrums. The peak occurs at a wavelength shift of $-0.14 \mathrm{~nm}$ $(-17.7 \mathrm{GHz})$, for $\Delta T=14{ }^{\circ} \mathrm{C}$ given the sensor sensitivity of $-0.8^{\circ} \mathrm{C} / \mathrm{GHz}$.

Note again the differential nature of the measurement: A baseline is recorded, and subsequent spectrum shifts indicate strain and/or temperature changes from the baseline state. A link to absolute temperature must be made by other means just as cold junction compensation is required for thermocouples. This baseline/calibration process is of particular concern since one will generally use a relatively small collection of point sensors to calibrate the distributed sensor. Difficulties in controlling and characterizing calibration accuracy along the entire length of a DTS grow with sensor length, but it is with long sensors that some of the major benefits of distributed sensing are realized.

DTS response is not determined solely by properties of the silica core and cladding, but also coatings applied to protect the waveguide. Durability and corrosion resistance are achieved through a wide variety of coatings and jackets, each with its own thermal expansion coefficient. Mismatches with silica can produce shear forces at the silica/ coating interface that generate tension or compression on the silica and perturb net temperature response of a sensor. In addition, coatings may be hygroscopic and swell with humidity increases, again generating shear forces and modifying sensor response. Humidity compensation may be necessary in some cases to achieve acceptable measurement accuracy. Humidity sensitivity was observed in the DTS employed for this study during transitions from ambient air to dry air from a compressor used to generate test flows. Section 5 covers DTS stability and humidity 
sensitivity to provide a sense of performance characteristics for measurements in flows.

\section{Experiment setup}

Applicability of the DTS for CFD validation data collection was evaluated in the MAX fluid dynamics facility at Argonne National Laboratory. Figure 6 shows an overview of the test section and instrument layout. Two air jets enter at the base of a $1 \times 1 \times 1.73 \mathrm{~m}$ long glass tank at atmospheric pressure. They impinge upon the lid and mix before flowing out a vent on the far side of the tank. The jet near the tank center is heated while the outer jet enters slightly below ambient temperature. The base and lid were fabricated from aluminum plates with a $920 \times 778 \mathrm{~mm}$ window milled out of the lid directly over the jets. A $50 \mu \mathrm{m}$-thick polyaramid film (Nomex ${ }^{\circledR}$ ) is stretched across the window for an infrared camera, which records surface temperature as it fluctuates in accordance with the movement and temperature changes of the jets underneath. The screen serves as the structure in the thermal fatigue scenario. Tank walls are made of transparent materials: soda-lime glass on the long sides and end panels of polycarbonate, which were machined for attachment of optical fiber support hardware. Flow for the jets is supplied by a compressor equipped with a refrigerated dryer that provides air at $\mathrm{RH}<10 \%$ at ambient conditions.

The optical fiber sensing system used for this study is an ODiSI (Optical Distributed Sensor Interrogator) model A50 from Luna Inc., configured to handle sensors up to $50 \mathrm{~m}$ in length with spatial resolution of $10 \mathrm{~mm}$ at data

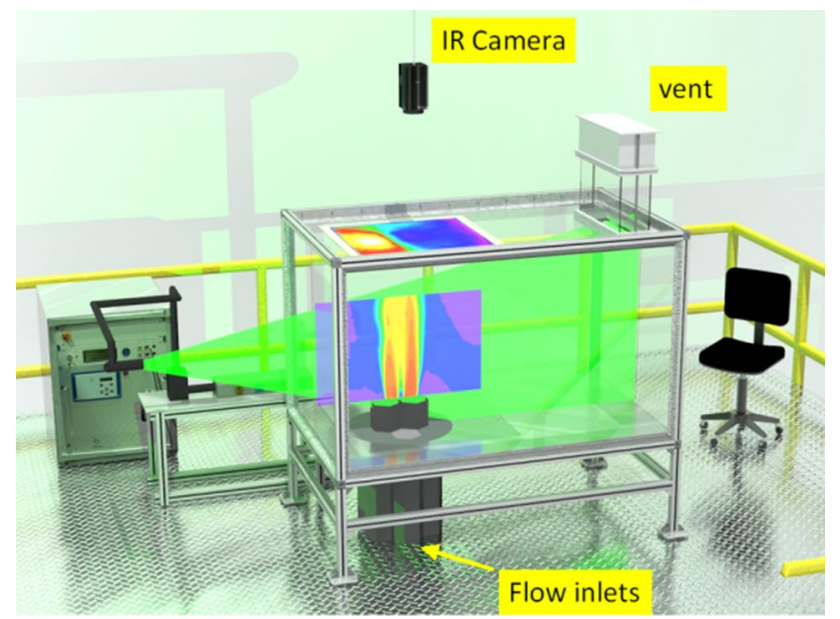

Fig. 6 Air jet-mixing experiments for thermal fatigue: air flows in through two hexagonal ducts and mixes before exiting through vent. Scalar contour within laser light sheet at tank midplane shows jet velocities; hot and cold jets impinge on polyaramid screen for temperature measurements by infrared camera rates up to $2.5 \mathrm{~Hz}$ and temperature spans to $\pm 1200{ }^{\circ} \mathrm{C}$ ( $\pm 10,000 \mu$ strain), though the sensor itself is likely to have a smaller service temperature range. The span for this study was set to $\pm 75{ }^{\circ} \mathrm{C}$, providing temperature resolution of $0.1{ }^{\circ} \mathrm{C}$ at a data rate of $2 \mathrm{~Hz}$. A later model interrogator, the ODiSI B (not used in this study), has a spatial resolution of $5 \mathrm{~mm}$ with a bandwidth of $250 \mathrm{~Hz}$ at a maximum sensor length of $2 \mathrm{~m}$ or $50 \mathrm{~Hz}$ for sensors up to $20 \mathrm{~m}$ long. Such data rates are more suitable for the turbulence spectrum expected of the jet-mixing tests, but far less flow field can be covered with these shorter sensors, and so the ODiSi $\mathrm{B}$ will be used in future studies for complementary measurements at higher temporal resolution. The ODiSI A50 covers the entire tank midplane and at data rates sufficient to illustrate implementation of a DTS in a CFD validation experiment.

\subsection{DTS layout}

The fiber optic sensors are made of $40 \mathrm{~m}$-long, $\phi 155 \mu \mathrm{m}$ polyimide-coated single-mode commercial telecom optical fiber (Specialty Photonics CL POLY 1310) that were tested for accuracy and repeatability to $150{ }^{\circ} \mathrm{C}$ (Lomperski et al. 2013). The optical fiber consists of a $\phi 125 \mu \mathrm{m}$ silica cladding around a $\phi 4.6 \mu \mathrm{m}$ silica core, with the latter effectively conveying the signals used for sensing. Figure 7 shows the mounting configuration with a single DTS extending across the tank midplane at sixteen levels. Spacing is $51 \mathrm{~mm}$ in the vertical direction, and the length at each level is $1.73 \mathrm{~m}$

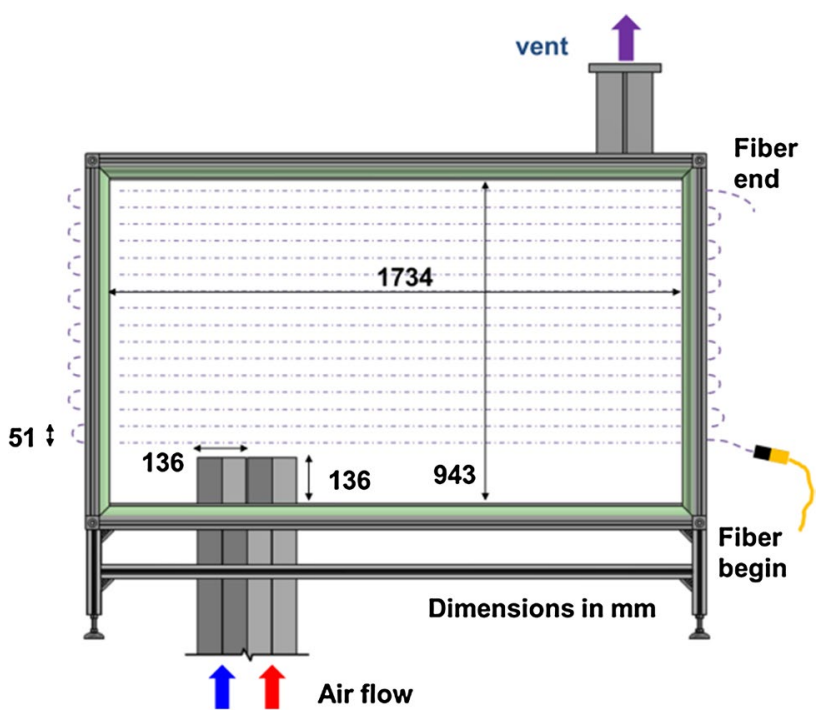

Fig. 7 Flow path and fiber configuration: 16 sections extend across tank midplane with DTS suspended from steel wire stretched between tank end plates. Bottom section is $38 \mathrm{~mm}$ from top of hexagonal ducts while the top section is $3 \mathrm{~mm}$ below the tank lid. The inner (right) jet is heated, while the outer jet is cooled slightly below ambient temperature by the compressor 


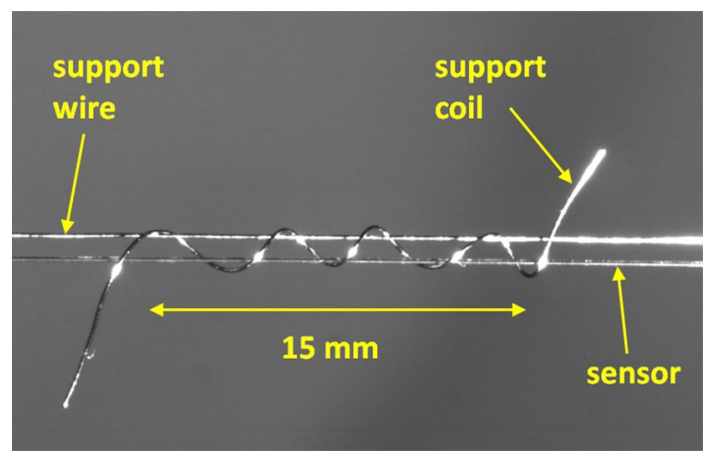

Fig. 8 Close-up of the DTS support configuration: $\phi 155 \mu \mathrm{m}$ sensor rests in coil suspended from wire pulled taut between tank end panels; both coil and support wire are $\phi 120 \mu \mathrm{m}$ stainless steel

for a total of $28 \mathrm{~m}$ within the tank for temperature measurements. Each $1.73 \mathrm{~m}$ span of optical fiber is denoted as a section. The bottom section is located $38 \mathrm{~mm}$ above the jet inlets and the top section $3 \mathrm{~mm}$ below the tank lid. The DTS passes through $1 \mathrm{~mm}$ holes in the walls to be wrapped around grooved Teflon disks that redirect it back into the tank for an adjacent section. Sections cannot be pulled taut in this configuration because the DTS responds to strain as well as temperature. Instead, they are hung from $\phi 120 \mu \mathrm{m}$ steel support wire stretched between the Plexiglas panels. Sections are suspended from this support with small coils $\sim 2 \mathrm{~mm}$ in diameter and $\sim 15 \mathrm{~mm}$ long made from the same $\phi 120 \mu \mathrm{m}$ wire (Fig. 8). Ten coils are distributed roughly equidistant along each section.

The tank expands slightly as it pressurizes from the $\Delta \mathrm{P}$ across a HEPA filter on the vent. To avoid DTS strain during expansion, slack is retained on one end of each section by draping a short portion $\sim 10 \mathrm{~mm}$ below the support wire. Slack is confined to $200 \mathrm{~mm}$ next to the wall furthest from the jets, a region of low flow velocity where the potential for flow-induced vibration and signal noise are minimal. Each region of slack is weighted with a small coil made of solder wire to provide constant tension that holds the rest of the section taut and alongside the support wire. This configuration introduces uncertainty in DTS location of approximately $\pm 5 \mathrm{~mm}$ along the draped regions, but temperature gradients there are low, and so this was considered acceptable for the purpose of this study.

\subsection{DTS baseline}

A baseline reading was recorded in stagnant ambient air after letting the tank sit overnight for more than $12 \mathrm{~h}$ to establish isothermal conditions. During the baseline, there was a $0.3{ }^{\circ} \mathrm{C} \Delta T$ between the inlet thermocouples near the bottom of the tank and vent thermocouples near the lid. This is sufficiently isothermal since our earlier study found DTS repeatability to be approximately $\pm 1{ }^{\circ} \mathrm{C}$ over the range of $20-150{ }^{\circ} \mathrm{C}$. It is assumed that humidity variations within the tank were negligible during the baseline.

Experiments are run with dry air from the compressor, making it necessary to compensate DTS signals for the apparent temperature shift that accompanies the humidity drop from baseline conditions. Humidity compensation was accomplished with a thermocouple at the cold inlet, which was compared with a short DTS segment directly above it. The thermocouple measured true $\Delta T$ between baseline and dry flow conditions (accuracy $\sim 0.1^{\circ} \mathrm{C}$ ), while the apparent $\Delta T$ from the DTS segment was recorded. The difference between the two corresponds to the DTS temperature offset due to the humidity change:

$\Delta T_{\Delta \mathrm{RH}}=\Delta T_{\mathrm{DTS}}-\Delta T_{\mathrm{TC}}$

The offset $\Delta T_{\Delta \mathrm{RH}}$ was applied to the entire DTS on the assumption that the humidity shift around the short DTS segment was duplicated throughout the tank. It was also assumed that humidity sensitivity is invariable along the length of the sensor. Note that the offset is roughly proportional to relative humidity, not absolute humidity, i.e., vapor pressure. Humidity response of the polyimide coating is considered in detail in Sect. 5.

A single-value correction presumes constant relative humidity during a test, which cannot coexist with nonisothermal conditions. Relative humidity is defined as the ratio of vapor partial pressure to saturation pressure at the local temperature:

$\mathrm{RH}=P_{\mathrm{v}} / P_{\text {sat }}(T)$

Under steady-state conditions, $P_{\mathrm{v}}$ is identical to the vapor pressure of the flow supplied by the compressor and equal throughout the tank. Therefore, relative humidity is necessarily lower in the hot jet than in colder regions because of the difference in saturation pressure. As a consequence, a single-value correction cannot generally be used for nonisothermal conditions. But it is sufficient in this case because the air is exceptionally dry, $\sim 5 \% \mathrm{RH}$, and the modest $\Delta T$ of $50{ }^{\circ} \mathrm{C}$ varies the humidity only $\sim 4 \% \mathrm{RH}$. DTS humidity sensitivity is roughly $0.2{ }^{\circ} \mathrm{C} / \% \mathrm{RH}$, leading to a maximum bias of approximately $0.8{ }^{\circ} \mathrm{C}$, which is confined to the hot jet. This was considered acceptable for this study since the bias is smaller than random shifts observed in an earlier stability study, noted below. Most of the DTS lies in cool regions where relative humidity remains close to the correction level.

The baseline and humidity compensation processes influence DTS accuracy, but they are not the sole determinants. Strain influences DTS response, and so measurement error depends to some extent on the physical configuration, e.g., how it is mounted within the test section and whether there is movement after baselining. The $\pm 1{ }^{\circ} \mathrm{C}$ repeatability 
cited above was measured for short lengths of optical fiber tested in an oven. DTS stability for the configuration shown in Fig. 7 has been examined in a separate study that measured drift for both stagnant and flow conditions (Lomperski and Gerardi 2014). The investigation found random shifts of less than $\pm 1{ }^{\circ} \mathrm{C}$ along most of the sensor. But shifts up to $\pm 2{ }^{\circ} \mathrm{C}$ were observed near the sensor end, and this larger value is conservatively adopted as the uncertainty estimate for the current study. Selected data from the stability study are presented in Sect. 5.

\subsection{PIV and ancillary instrumentation}

The main components of the PIV system are a Litron LDY303 Nd:YLF dual cavity pulsed laser and an IDT Y7 camera equipped with a $1920 \times 1080$ array of $7.24 \times 7.24 \mu \mathrm{m}$ pixels. The field of view used here was $240 \times 425 \mathrm{~mm}$ taken with a Nikon $35 \mathrm{~mm}$ lens at an aperture of 2. Image pairs with a $\Delta t$ of $2500 \mu$ s were recorded at $20 \mathrm{~Hz}$. Pulse power was $30 \mathrm{~mJ}$ and light sheet thickness approximately $2 \mathrm{~mm}$. IDT's Motion Studio was used for both laser and camera triggering and image capture. Analysis was performed with Dantec's Dynamic Studio using adaptive correlation with $32 \times 32$ pixel interrogation areas $(7 \times 7 \mathrm{~mm})$ with $50 \%$ overlap and a subpixel refinement option. The resulting vector field was not filtered, but range validation was used to eliminate physically unrealistic data.

Flow was seeded with a polyethylene glycol mist at an average droplet diameter of $\sim 2 \mu \mathrm{m}$. Mist was introduced at the bottom end of the flow channels, $2.7 \mathrm{~m}$ from the tank, resulting in a particularly homogenous seeding density. Mist flow rate to each channel was $35 \mathrm{slpm}$, measured with Teledyne model 301 thermal mass flow meters to an accuracy of $\pm 0.4 \mathrm{slpm}$, while the main flow to each jet was measured with Teledyne 307 flow controllers to within \pm 8 slpm.

The infrared camera is a Cedip Infrared Systems Silver $660 \mathrm{M}$ with a $640 \times 512 \mathrm{InSb}$ (indium antimonide) focal plane array operating in a spectral range of 3-5 $\mu \mathrm{m}$ through a $27 \mathrm{~mm}$ lens. The field of view was $550 \times 440 \mathrm{~mm}$ for a spatial resolution of $0.86 \mathrm{~mm} /$ pixel. Accuracy is $\pm 1{ }^{\circ} \mathrm{C}$ over the temperature range used here. Measurements were made at $2 \mathrm{~Hz}$ over a period of $600 \mathrm{~s}$ for a record length of 1200 frames.

\section{Results}

\subsection{DTS data}

We present here flow field measurements for the following boundary conditions: cold jet inlet at $22{ }^{\circ} \mathrm{C}$ and hot inlet at $70{ }^{\circ} \mathrm{C}$ with flow rates of 535 and 335 slpm, respectively; cold inlet velocity of $0.56 \mathrm{~m} / \mathrm{s}$ with a Reynolds number of 4900 , hot inlet at $0.35 \mathrm{~m} / \mathrm{s}$ and 2200 . At these velocities, perturbation of the flow field by the DTS is slight. The Reynolds number at the $\phi 155 \mu \mathrm{m}$ fiber peaks near $R e=6$ (or $R e \sim 12$ if the $\phi 120 \mu \mathrm{m}$ wire support is included as well) directly over the cold inlet and also midway along the accelerating hot jet. It is much lower everywhere else. Flow separation behind a cylinder begins near $R e=5$, but eddies remain attached until $R e \sim 40$. Therefore DTS-generated turbulence is expected to be negligible.

Flow rates were chosen to establish roughly similar inlet velocities while minimizing signal noise associated with flow-induced vibration. Noise was observed to increase with inlet velocity between 0.5 and $2 \mathrm{~m} / \mathrm{s}$, and so the lower end of the range was targeted to minimize measurement uncertainty. All measurements were made during a steady state that followed a $6 \mathrm{~h}$ heat-up stage to bring structures to thermal equilibrium.

Figure 9 shows raw data from a single scan of the $40 \mathrm{~m}$-long sensor plotted as measured temperature shift from the baseline condition. The first $10 \mathrm{~m}$ remained on the spool outside the tank at uniform temperature, while the rest was either in the tank or outside on the Teflon support disks. The plot shows 16 peaks, one for each section where it passes over the hot jet. The signal dips near zero where the sensor is outside the tank for the U-turn that brings it to a new section and level.

Raw data must be decomposed to isolate the sections suspended within the tank. Section endpoints were identified in advance of the flow test when the tank was open for access to the DTS. The sensor was touched briefly with a hot soldering iron at each point where it met the tank wall, generating a temperature spike. The transient was registered by the sensing system to produce a signal similar to that of Fig. 9, but with a single peak. The center of the peak corresponded to the end of a section. The collection of 16 sections matches in length within $10 \mathrm{~mm}$, and so uncertainty in position is estimated at approximately $\pm 5 \mathrm{~mm}$.

Figure 10 presents the decomposed temperature data for selected sections, each consisting of 175 data points at $10 \mathrm{~mm}$ intervals using a $30 \mathrm{~mm}$ gauge length. The origin is located at the bottom of the tank between the two inlets to give a side view perspective coinciding with Fig. 7. The position of the hot jet over the inlet is apparent along with a broadening of its perimeter and decline in peak temperature in the direction of the tank lid. The adjacent cold jet is visible as a slight depression that persists in all but the uppermost sensor section. There is also a slight shift of the cold jet toward the nearside wall that increases with height. Outside the jet region, temperature generally increases with elevation. The temperature profile changes little with elevation until it reaches the top section located $\sim 3 \mathrm{~mm}$ below the lid, where mixing depresses and markedly broadens 


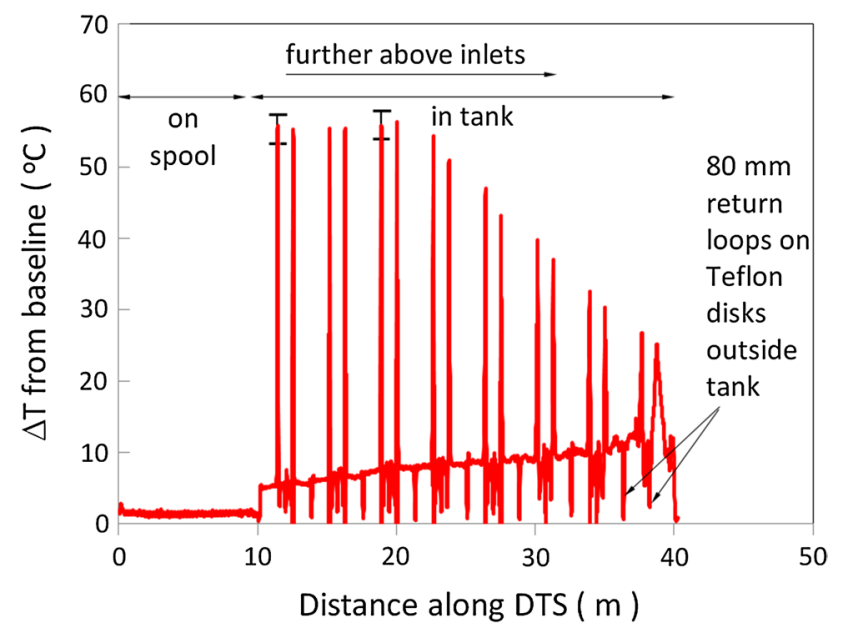

Fig. 9 A single scan of the DTS from a $2 \mathrm{~Hz}$ data stream; hot inlet flow at $70{ }^{\circ} \mathrm{C}$ and cold inlet at room temperature; peaks occur where sensor is directly above hot jet

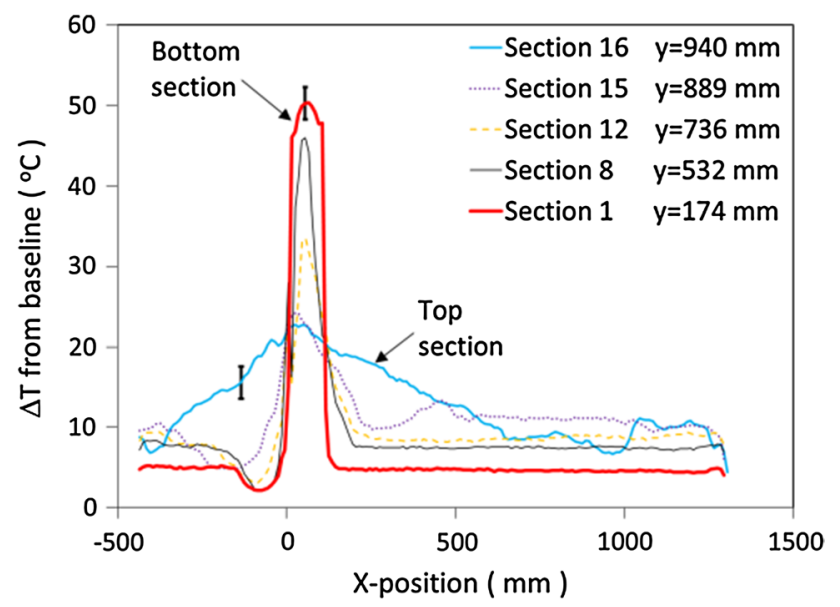

Fig. 10 Measured temperature shift from baseline condition; signal shown in previous figure decomposed to locate sections within tank; 175 data points/section. Origin is centered between inlets

the peak. Section 15 exhibits a dip next to the hot jet near $x=250 \mathrm{~mm}$ that is inconsistent with the other sections. After falling to a minimum, it rises with distance from the jet instead of exhibiting a steady decline (section 16) or remaining flat (all other sections). This appears to be a stable cold air pocket $50 \mathrm{~mm}$ below the lid. Though seemingly unlikely, a similar, milder dip and recovery occur in section 14 (not shown), and so the measurement appears reliable.

The DTS source data were converted to absolute temperature for plotting of all sixteen sections as a $2 \mathrm{D}$ contour across the tank midplane using all 2770 data points (Fig. 11). The plot is based on an average of 1200 scans recorded at $2 \mathrm{~Hz}$. Resolution is $10 \mathrm{~mm}$ in the horizontal

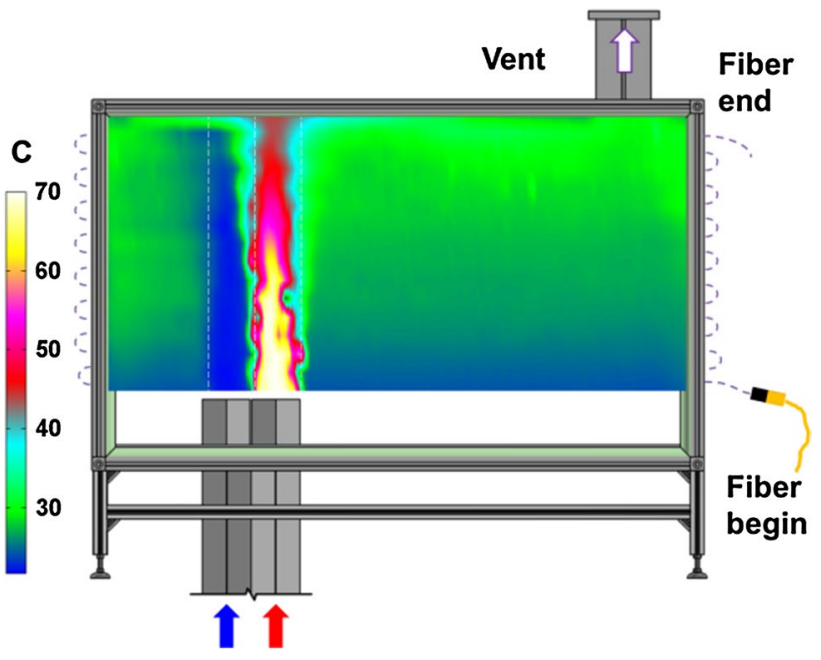

Fig. 11 Temperature across tank midplane based on all 16 fiber sections; 2770 data points at $10 \mathrm{~mm}$ spacing derived from $30 \mathrm{~mm}$ gauge length; linear interpolation across $51 \mathrm{~mm}$ vertical span between sections

direction and $51 \mathrm{~mm}$ in the vertical with linear interpolation between sections. Despite coarse resolution in the vertical direction, the DTS provides a clear sense of plume location and the extent of thermal mixing. The core of the hot jet and its dissipation in the vertical direction are distinctly visible. Temperature resolution is sufficient to observe the outline of the cold plume though it is only a few degrees colder than neighboring, well-mixed regions. The majority of the tank atmosphere is well-mixed with temperatures in the range of $25-30{ }^{\circ} \mathrm{C}$. The vertical temperature gradient in the mixed regions is remarkably similar on each side of the plume despite differing distances to the tank end walls. It would be reasonable to expect distinctly lower temperatures in the region between the cold jet and wall, but this is not observed. Note, though, that asymmetries may exist away from the midplane, and regions of poorly mixed flow could travel across the lid and downward against the walls of the tank. Additional sensors distributed across the tank could potentially reveal such asymmetries.

\subsection{Infrared and PIV data}

Figure 12 shows average polyaramid screen temperature measured with the infrared camera. Outlines of the hexagonal inlets are drawn for orientation to the side view of the previous figure. The contour is largely symmetric, and peak temperature nearly centered over the hot inlet with a $15 \mathrm{~mm}$ offset from the midplane. The symmetry might suggest that only the hot jet is active, but boundary conditions are identical for all data presented here in Sect. 4, with cold and hot inlet flow rates of 535 and 335 slpm, respectively. The green cross on the figure marks peak 


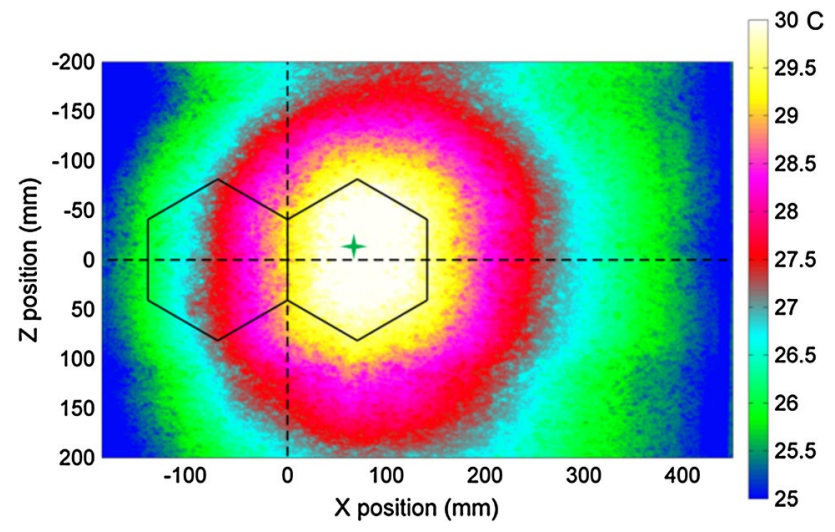

Fig. 12 Top view of screen/lid as seen by IR camera: average temperature over $600 \mathrm{~s}$ recorded at $2 \mathrm{~Hz}$; green cross marks the peak temperature. Cold jet is active, but the buoyant hot jet stratifies and heats the lid with unexpected symmetry

screen temperature. This same peak location was found in a data set recorded just prior at a rate of $1 \mathrm{~Hz}$ for $1200 \mathrm{~s}$, suggesting that the plume is stable. The RMS data (standard deviation $\sigma_{\mathrm{T}}$ ) exhibits the same symmetric pattern seen here for temperature: screen temperature and $\sigma_{\mathrm{T}}$ peak at 30 and $0.5^{\circ} \mathrm{C}$, respectively.

Figure 13 shows the velocity field in the vertical plane directly beneath the tank lid based on an average of 4000 image pairs recorded for $200 \mathrm{~s}$ at $20 \mathrm{~Hz}$. The map of vertical velocity component in Fig. 13a provides an indication of jet position below the lid. The hot jet enters the region pictured at a peak velocity of $\sim 0.8 \mathrm{~m} / \mathrm{s}$ while the cold jet is near $0.5 \mathrm{~m} / \mathrm{s}$. Figure $13 \mathrm{~b}$ reveals a stagnant region with flow moving laterally along the underside of the screen away from the stagnation point. The result is not self-evident since there might be several stable stagnant regions or none, and stagnation points can be located off the centerline. It is interesting to note that the hot jet has accelerated from the inlet condition of $0.35 \mathrm{~m} / \mathrm{s}$ while the cold jet slowed slightly. This suggests that buoyancy forces have a strong influence on the flow field despite the modest jet $\Delta T$ of $50^{\circ} \mathrm{C}$. The relative strength of buoyancy and inertial forces is represented by the Richardson number, which can be written in terms of the Grashof and Reynolds numbers:

$R i=G r / R e^{2}$

A Grashof number of $5 \times 10^{9}$ is obtained using the tank height of $1 \mathrm{~m}$ and $\Delta T$ of $\sim 50{ }^{\circ} \mathrm{C}$ between the hot jet and mixed regions. The hot jet Reynolds number is 2200 , resulting in $R i \approx 1000$ and confirmation of buoyancydriven flow.

Note that the PIV data plane is displaced $20 \mathrm{~mm}$ from the DTS plane at the tank centerline to avoid the sensor supports, which would interfere with the laser sheet. This sort of conflict is a drawback of fiber optic sensing versus
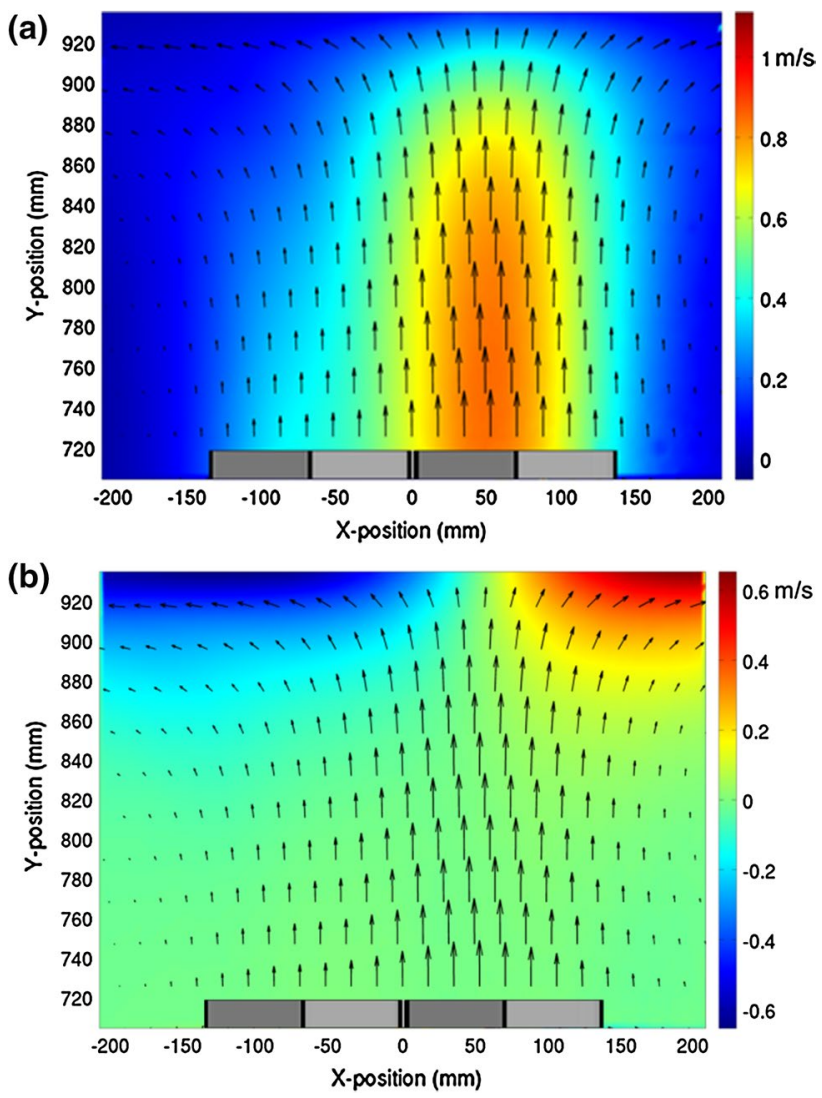

Fig. 13 Measured velocity in the vertical plane directly below screen displaced $20 \mathrm{~mm}$ off tank midplane; color contours show a vertical component and $\mathbf{b}$ horizontal component. Hexagonal inlets end at $y=136 \mathrm{~mm}$, but are included as an orientation aid

a purely optical technique such as LIF, which can often be used along with PIV in the same plane. And moving a DTS across the tank to sample other regions can be a delicate matter. A LIF data plane could be shifted more easily since the laser and camera would be outside the tank. Still, a DTS requires no powerful laser, camera or chemical seeding, and it functions equally well in opaque and transparent fluids.

\subsection{Data reduction for thermal fatigue application}

Thermal cycling is driven by temperature transients in the flow field and heat transfer with the adjacent structure. CFD validation requires flow field temperatures and velocities along with structure temperatures and a complete set of boundary conditions. We provide here a simple example of the role of the DTS in such a data set.

The polyaramid screen serves as the structure of interest with temperature determined via the IR measurements of Fig. 12. Gas temperature and velocity were measured on vertical planes, and so data at the uppermost level of each are compared with a line sample of IR data along 


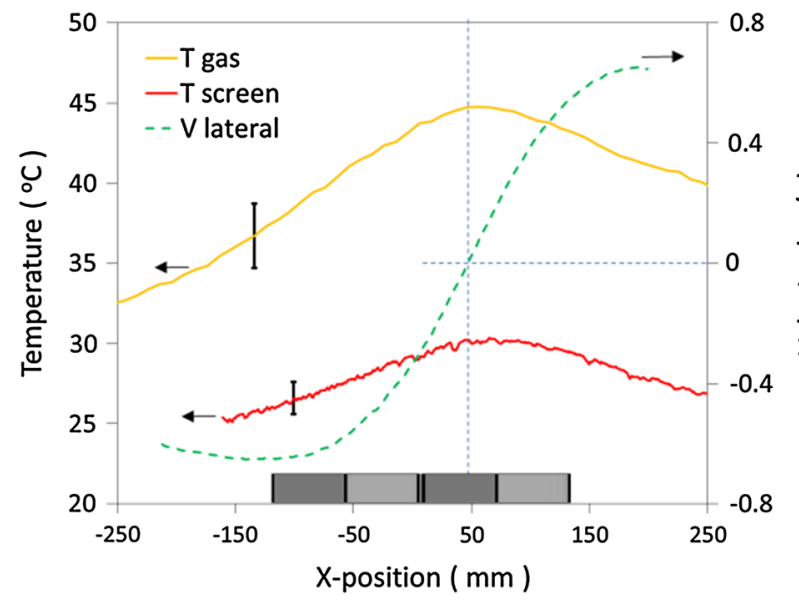

Fig. 14 Screen temperature, gas temperature and velocity along three proximate lines: screen and gas temperatures along tank midplane with latter $3 \mathrm{~mm}$ below screen, velocity directly below screen $20 \mathrm{~mm}$ from midplane

the screen centerline. This constitutes the required set of temperature and velocity along nearly the same line. The data are plotted in Fig. 14 with the inlets shown for reference. The plot shows that screen and gas temperatures peak in approximately the same location, but not quite at the stagnation point. Peak temperatures are nearly centered over the hot inlet, while the stagnation point is offset by $30 \mathrm{~mm}$.

Structure temperature history is the ultimate concern for the fatigue application, with interest often focusing on frequency spectrums. Moderate frequencies are generally of greatest import since high-frequency temperature fluctuations do not penetrate deeply into structures. Frequency analysis is beyond the scope of this paper, but we include RMS plots of temperature and velocity as they are of use in code validation even in absence of cyclic flow behavior. Figure 15 shows temperature RMS peaking near the center of the hot inlet, while velocity RMS lacks any clear pattern. It is known from the 2D IR data that peak temperature and RMS are slightly off the midplane. A more comprehensive data set for thermal striping would include gas temperature and velocity measurements across the entire underside of the screen rather than just at the midplane. This could be accomplished by reorienting both the PIV and DTS measurement planes into the horizontal plane.

\section{DTS measurement uncertainty}

Temperature measurement uncertainty stems from two distinct sources: the electronics and the sensor itself, with most residing in the latter. Repeatability of the A50 interrogator is $\pm 0.2{ }^{\circ} \mathrm{C}$, which is adopted here as the total uncertainty

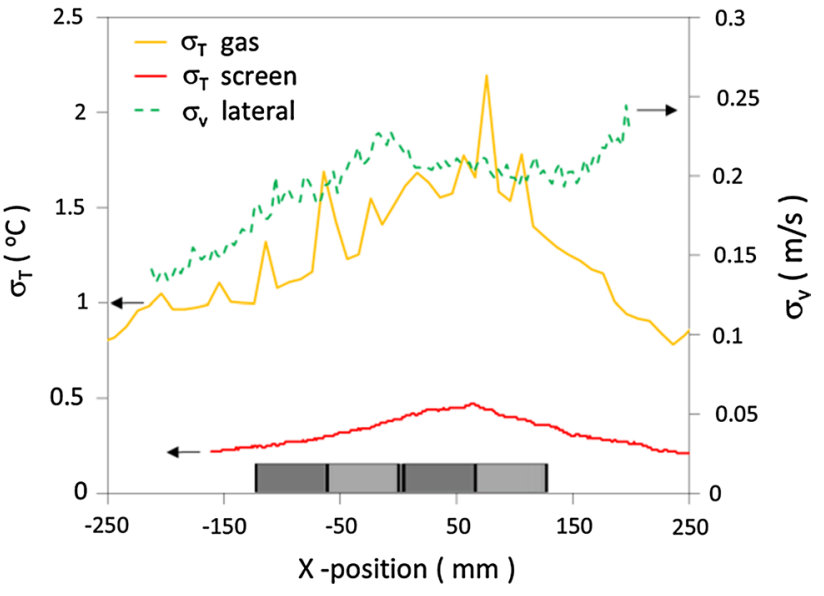

Fig. 15 RMS for screen temperature, gas temperature and velocity

associated with the electronics. For the sensor, uncertainties lie in the temperature coefficient of the fiber optic cable (linearity and accuracy limits of $K_{\mathrm{T}}$ in Eq. 6, including hysteresis), the baseline process, and humidity and strain variations after baselining. Strain can develop through static or dynamic loading by the flow field or movement of support structures. An estimate of total temperature measurement uncertainty is obtained via the standard error propagation formula:

$\sigma_{\mathrm{T}}^{2}=\sigma_{K_{\mathrm{T}}}^{2}+\sigma_{b}^{2}+\sigma_{\mathrm{RH}}^{2}+\sigma_{\varepsilon}^{2}$

Uncertainty in $K_{\mathrm{T}}$ was assessed in a previous study that examined sensors held within a small oven (Lomperski et al. 2013). Repeatability was within $\pm 1{ }^{\circ} \mathrm{C}$ over three cycles between room temperature and $150{ }^{\circ} \mathrm{C}$, and this figure is adopted for $\sigma_{\mathrm{KT}}$. Strain was avoided in these tests by encasing sensors within stainless steel capillaries.

Baseline uncertainty $\sigma_{\mathrm{B}}$ arises from tank temperature and humidity variations at the moment the baseline is recorded, with the former estimated at $\pm 0.3{ }^{\circ} \mathrm{C}$ (the observed $\Delta T$ between TCs at tank top and bottom during baseline), and the latter considered negligible. Uncertainty due to post-baseline shifts in humidity, $\sigma_{\mathrm{RH}}$, is less than $\pm 0.8{ }^{\circ} \mathrm{C}$ as noted earlier in describing the baseline procedure. The final term $\sigma_{\varepsilon}$,uncertainty linked to strain changes after baselining, is estimated to be $<2{ }^{\circ} \mathrm{C}$ for a total temperature uncertainty $\sigma_{\mathrm{T}}$ of approximately $\pm 2.4{ }^{\circ} \mathrm{C}$. The basis for $\sigma_{\varepsilon}$ is detailed in the next section. Note that the uncertainty sources identified here are not necessarily random and so Eq. 10 provides only an estimate of total measurement uncertainty.

Dominance of the strain term in Eq. 10 originates from our sensor support configuration: bare fiber optic cable suspended in a fashion that permits movement and hence strain. If, instead, the sensor was placed within a steel 
capillary, $\sigma_{\varepsilon}$ would be negligible. The sensor would also be isolated from humidity variations. As a result, $\sigma_{\mathrm{T}}$ would be the same $\pm 1{ }^{\circ} \mathrm{C}$ found in the oven repeatability test. But the bare fiber configuration was chosen over the capillary to minimize thermal response time.

The response time of a $\phi 500 \mu \mathrm{m}$ steel capillary is an order of magnitude higher than that of a bare fiber for the flow conditions used here. For example, at a cold jet inlet velocity of $0.5 \mathrm{~m} / \mathrm{s}$, the heat transfer coefficient in crossflow is $\sim 250 \mathrm{~W} / \mathrm{m}^{2} \mathrm{~K}$ for the bare fiber and $\sim 125 \mathrm{~W} / \mathrm{m}^{2} \mathrm{~K}$ for the capillary, the latter being lower on account of the larger diameter. In both cases, the Biot number is $\ll 1$, and so a simple lumped parameter model can characterize time response: $\sim 0.25 \mathrm{~s}$ for the bare fiber and $\sim 4 \mathrm{~s}$ for the capillary. Response time is superior for the bare fiber at the expense of higher measurement uncertainty. Note that thermal response is considerably faster in higher-conductivity fluids: $\sim 0.02 \mathrm{~s}$ for the same $\phi 500 \mu \mathrm{m}$ steel capillary in $0.5 \mathrm{~m} / \mathrm{s}$ water flow.

Though fast response may not be necessary for many applications, high bandwidth is generally valued in CFD validation experiments and so there is strong incentive to minimize sensor mass. The remainder of this section summarizes our assessments of strain-induced drift and humidity sensitivity to provide a better understanding of the basis of the above error estimates. This additional context is warranted since DTS sensitivity to handling and humidity is quite unlike conventional temperature sensors such as thermocouples and RTDs.

\subsection{Drift in stagnant conditions}

A previous study examined a collection of nine sensors for accuracy and repeatability between ambient temperature and $150{ }^{\circ} \mathrm{C}$ using a small oven (Lomperski et al. 2013). The controlled environment isolated sensor temperature response by eliminating the role of strain. A slightly nonlinear response was observed with repeatability within $\pm 1{ }^{\circ} \mathrm{C}$ over three cycles between room temperature and $150{ }^{\circ} \mathrm{C}$. Such tests cannot, however, completely characterize accuracy, stability and repeatability for flow applications because sensors respond also to strain. Overall accuracy ultimately depends in part upon application specifics such as mounting configuration and mobility during measurements. The basis for $\sigma_{\varepsilon}$ is of particular interest because it is the dominant term in Eq. 10 for the configuration used here, thus we have conducted separate tests to isolate and assess random errors associated with strain.

Sensor stability was examined under stagnant conditions at room temperature over the course of 1 week in the physical configuration shown earlier in Fig. 7. The support configuration is the same as that described in Sect. 3 but with two $40 \mathrm{~m}$ DTSs rather than just one. The two sensors traverse identical paths and are in close physical contact, a key point since stability is examined via the $\Delta T$ between sensors.

Thermocouples were used to monitor temperature at the tank inlets and vent, and a hygrometer at the bottom of the tank for humidity. The vent and inlets were sealed to slow humidity shifts associated with variations in ambient humidity. The vent thermocouples were inside the tank gas space, but inlet TCs were $150 \mathrm{~mm}$ below Teflon plugs that covered the inlets to isolate the tank. After sealing the tank, it sat undisturbed for $7 \mathrm{~h}$ before recording DTS baselines to serve as reference states. Figure 16 shows thermocouple and hygrometer data, which varied less than $1{ }^{\circ} \mathrm{C}$ and $7 \% \mathrm{RH}$, respectively, over the course of the 7-day test. The exceptional temperature stability is due to the unusual nature of the lab space, a concrete containment with $1 \mathrm{~m}$-thick walls. The intermittent temperature spikes occurred when room lighting was switched on to log data. The offset between the vent and inlet TCs is stable, and paired TCs remained within $0.1{ }^{\circ} \mathrm{C}$ of one another over the entire 7-day span. The TC data demonstrate stability of the tank atmosphere while also providing a benchmark for DTS stability.

DTS stability was assessed by examining $\Delta T(x)$ between the two sensors as a function of time, where $\Delta T(x)=T_{1}(x)-T_{2}(x)$. Figure 17 shows $\Delta T$ for three selected DTS sections: bottom, middle and top levels out of the total of sixteen. Data span the entire length of each section from one tank wall to the other. Each curve represents $\Delta T$ along a section on a single day. Data are based on an average of 100 readings logged at $1 \mathrm{~Hz}$ with a gauge length of $20 \mathrm{~mm}$ and spacing of $3 \mathrm{~mm}$ for a total of 580 data points/section. The plot uses moving spatial averages along the sections to improve clarity and ability to discern data sets from different days.

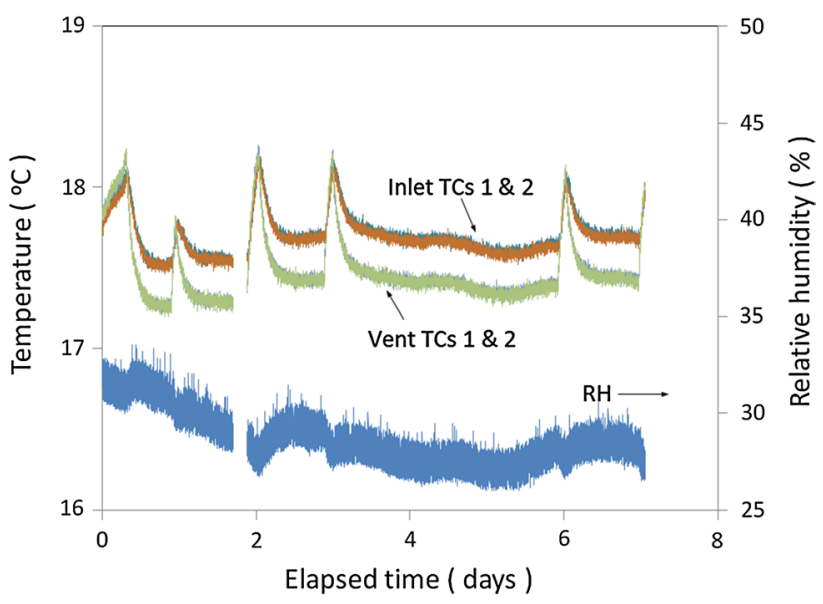

Fig. 16 Tank humidity and temperature over course of stability test under stagnant conditions with inlets and outlet sealed; temperature spikes caused by ambient lighting switched on to log measurements 


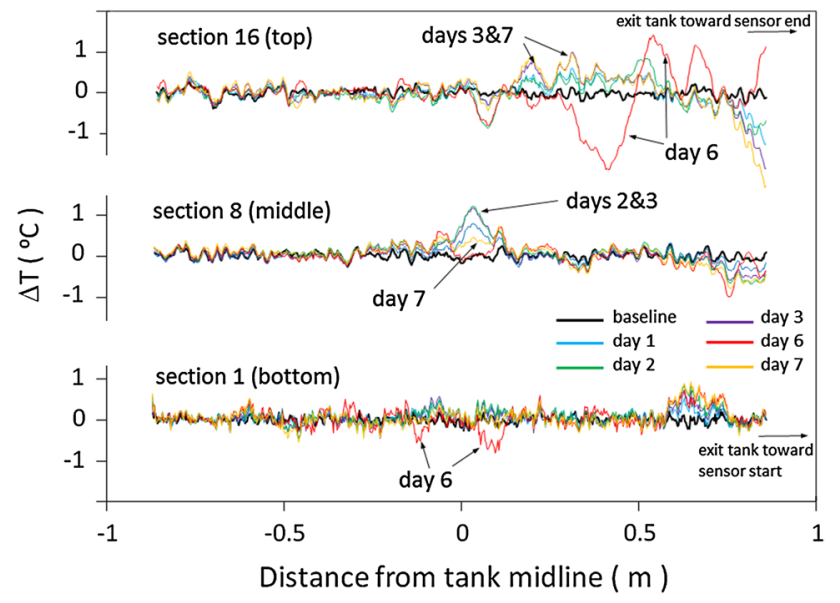

Fig. 17 Stagnant conditions: $\Delta T$ between a pair of parallel DTSs mounted as shown in Fig. 7. Sections at top, middle and bottom levels of tank selected as examples of test results; baselines in black

Absolute temperature is not the figure of merit because complete temperature and humidity uniformity for the baseline could not be ensured. Baselines would incorporate any initial spatial variations in temperature so that later changes, even progression toward a more uniform atmosphere, would appear as unexplained drifts in absolute temperature. The $\Delta T$ is used instead since the baselines are recorded at nearly the same time, one immediately after the other, with essentially the same temperature irregularities. Subsequent local changes in tank temperature or humidity should be echoed by both sensors so that, ideally, $\Delta T$ remains zero (within deviations of the baseline) along the entire sensor tandem. These stagnant, isothermal conditions present the least demanding test of sensor stability.

Figure 17 shows that over the course of 7 days $\Delta T$ generally remained close to the baseline and well below $0.5^{\circ} \mathrm{C}$. The most notable exception is the far end of the top section, which exhibits shifts up to $\sim 2{ }^{\circ} \mathrm{C}$. This region presents a special case where sensors exit the tank and ends are taped outside to the glass, possibly allowing transmission of strain back to the section in the tank. Such large shifts did not occur for section 1, which also leads out of the tank, but there sensors were directed to spools storing excess fiber. Boundary conditions for sections 1 and 16 were similar, but not identical.

We had expected $\Delta T$ to be comparable with the repeatability of TCs since the sensors were in close proximity and the tank atmosphere nearly isothermal with only slow drifts in temperature and humidity. But drift across isolated regions like that near the midpoint of section 8 was observed in other segments as well (not shown). The source of drift remains unclear, but it is within the $\pm 1{ }^{\circ} \mathrm{C}$ limits measured in the oven test. Each section retained slack to prevent tension changes from any movement of tank walls. Relative movement between DTSs was unlikely as they were in close contact and stuck together slightly due to a thin layer of polyethylene glycol deposited from mist used for PIV. The sensors are insensitive to polyethylene glycol, but it may lead to local variations in the rate at which water vapor can diffuse in or out of the polyimide coating to achieve equilibrium. Or drift may be associated with tension changes from support coil movement, but it is difficult to conceive of significant strain shifts since the optical fibers are quite light. Still, the fibers are also very thin and the strain equivalent of $1{ }^{\circ} \mathrm{C}, 8 \mu$ strain, can be generated by the weight of a paperclip.

\subsection{Flow-induced shifts}

The 7-day stability study examined DTS behavior under stagnant conditions as a presumed best case for straininduced drift with our support configuration. A second set of $\Delta T$ measurements was recorded after subjecting sensors to isothermal jet flow (Fig. 18). The data are again for stagnant conditions. Dynamic pressure on the sensor from the flow is low and associated offsets negligible. For example, an offset of $\sim 0.06{ }^{\circ} \mathrm{C}$ is observed at $0.5 \mathrm{~m} / \mathrm{s}$ for a sensor stretched across the inlets.

Data for day 8 were recorded after testing at jet Reynolds numbers of 2700,3800, and 5500; day 9 data followed tests at $\operatorname{Re}=7100,8200$, and 9300. The new $\Delta T$ data exhibit larger shifts than pre-flow test data, but generally remain within a window of $\pm 1{ }^{\circ} \mathrm{C}$. Pronounced variations are again seen near the end of segment 16 where the sensors exit the tank.

On average, drift from the baseline increased slightly beyond that observed before flow testing. But the new

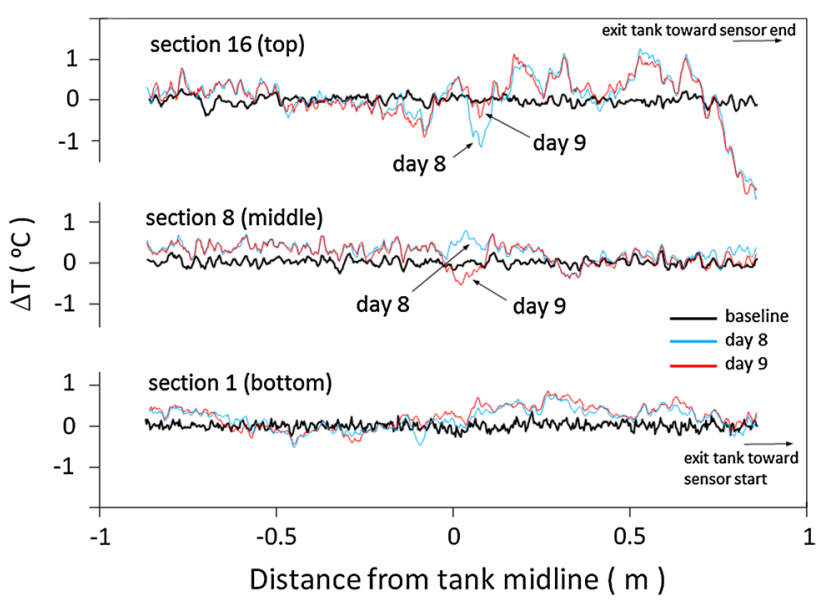

Fig. 18 Stagnant conditions: $\Delta T$ between DTSs after flow tests. Day 8 recorded after testing at Reynolds numbers up to 5500; day 9 testing up to $\operatorname{Re}=9300$. Baselines in black same as those of Fig. 17 
shifts have a different character from those of the pre-flow data set, with consistent shifts extending across several support coils (spacing $\sim 150 \mathrm{~mm}$ ) rather than just short, isolated zones. A constant offset would be expected if residual strain remained in one sensor after shifting within the array of spring supports during the flow phase.

The post-flow data suggest stability roughly equivalent to that observed in the thermal cycling study with the oven, but this type of testing cannot directly indicate stability while in an active flow. Instead, it provides a sense of the best that can be expected for the tested flow rates using this particular support configuration. A preliminary assessment of accuracy in isothermal jet flow can be found in (Lomperski and Gerardi 2014).

\subsection{Humidity response}

The fiber optic sensors used for this study are made of $\phi 155 \mu \mathrm{m}$ polyimide-coated single-mode commercial telecom fiber (Specialty Photonics CL POLY 1310). Polyimide is hygroscopic and swells with an increase in humidity (Bhargava et al. 2006), an effect exploited to create fiber Bragg grating humidity sensors (Ding et al. 2011; Berruti et al. 2013). Swelling of the coating alters optical fiber dimensions and shifts the Bragg wavelength. Similar shifts could be expected for the DTS used here since its functional principle is analogous to a continuous series of weak Bragg gratings. We observed signal shifts in our experiments after taking baselines with ambient air and afterward switching to flowing conditions with air supplied by a compressor. Shifts up to $-9{ }^{\circ} \mathrm{C}$ were observed despite little actual temperature change from the stagnant state. This was later linked to humidity changes since the compressor is equipped with a dryer that reduces humidity to $<10 \% \mathrm{RH}$, well below typical ambient levels.

We have recorded a set of humidities and DTS signal shifts to provide a sense of apparent temperature changes that can accompany ordinary changes in room humidity. The data give an indication of the level of climate control necessary to achieve target sensor stability and measurement accuracy. Two DTSs were placed in a 1 liter steel container with a thermocouple and a Dwyer series RHP humidity monitor (accuracy $\pm 2 \% \mathrm{RH}$ ). One was the POLY 1310 fiber used for our mixing experiments and the other a gold-coated optical fiber. The former is $3 \mathrm{~m}$ long and the latter $2 \mathrm{~m}$, both wound on $\phi 90 \mathrm{~mm}$ spools. The sensors were baselined at ambient humidity, and afterward, humidity was varied and the signal shift recorded; they were given at least $1 \mathrm{~h}$ to reach equilibrium. Results are plotted in Fig. 19, which shows a lack of humidity sensitivity for the gold-coated sample and an approximately linear change of $\sim 0.2{ }^{\circ} \mathrm{C} / \% \mathrm{RH}$ for the polyimide-coated sample. One might expect sensitivity to vary linearly with vapor

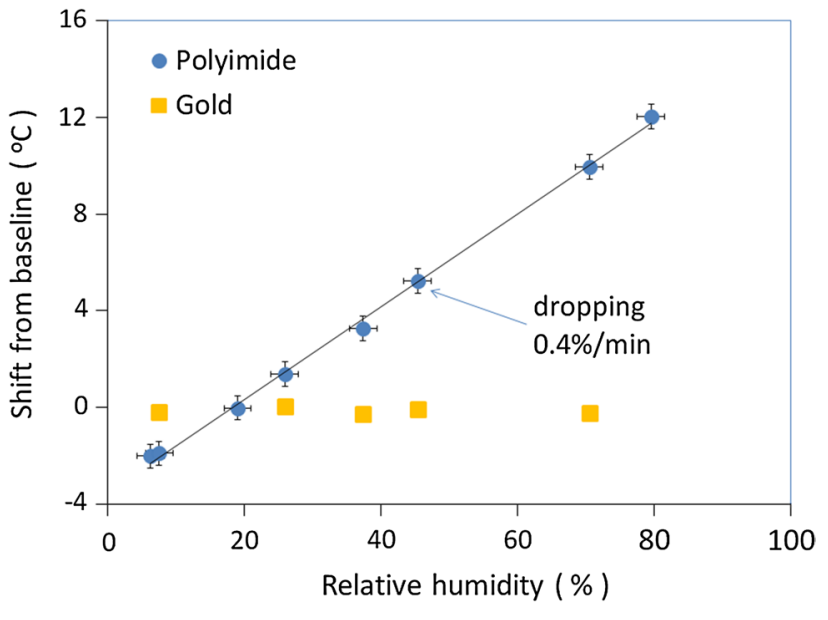

Fig. 19 Measured temperature shift versus humidity for a goldcoated and a polyimide-coated sensor. All data at steady state except point noted

pressure (absolute humidity) rather than relative humidity, but Buchhold et al. (1998) demonstrated that strain in polyimide films varies nearly linearly with relative humidity. Indeed, fiber Bragg grating humidity sensors exhibit a linear dependence of wavelength shift on relative humidity (Ding et al. 2011; Berruti et al. 2013).

The low end of the humidity spectrum in Fig. 19 corresponds to compressor air. Shifts up to $\sim 10{ }^{\circ} \mathrm{C}$ can occur with our setup after baselining at ambient humidity. Humidity sensitivity may be of concern even in applications involving only ambient air since, for example, significant humidity changes can accompany a passing weather front. Metal-coated fibers lack humidity sensitivity, but the gold-coated fiber is custom-made and more costly than commercial telecom fiber, and its stability under thermal cycling is inferior to that of the polyimide-coated fiber (Lomperski et al. 2013).

Note that the shift data presented here are not broadly applicable to all polyimide-coated optical fibers since absorption characteristics can vary with the chemical structure of the polyimide as well as processing parameters (Buchhold et al. 1998). In addition, film thickness influences the humidity response of fiber Bragg sensors (Berruti et al. 2013), and so this can be expected also for distributed fiber optic sensors. An ordinary humidity compensation scheme may be sufficient for some applications, but potentially problematic for high, or highly variably humidity since it influences film/substrate adhesion strength (Hu and Chen 1992). Adhesion characteristics influence mechanical interaction between optical fiber coating and cladding during temperature change, tension, and coating swelling. Inconsistent coupling is likely to lead to drift or shifts in sensor response and degradation of repeatability and accuracy. 


\section{Discussion}

The DTS generates thousands of temperature measurements across a relatively large flow field without need of any optical access. Though spatial and temporal resolution does not match that of LIF systems, the DTS delivers data density far greater than anything achievable with traditional sensors like thermocouples, and without cameras and high-power lasers. Fiber optic sensors may interfere with PIV and are less mobile than LIF light sheets, but they can wrap around complex surfaces for 3D temperature mapping that would be difficult to achieve with planar LIF measurements. Recent advances have increased electronics bandwidth to $250 \mathrm{~Hz}$, adequate in many cases to characterize turbulence spectrums. However, the sensor itself will ultimately limit bandwidth via heat transfer rates between optical fiber and flow field. Thermal response time of the $\phi 155 \mu \mathrm{m}$ sensor used in this study is $\sim 0.25 \mathrm{~s}$ when immersed unclad in $0.5 \mathrm{~m} / \mathrm{s}$ air flow and $\sim 4 \mathrm{~s}$ when sheathed in a $\phi 500 \mu \mathrm{m}$ steel capillary. Response time for the capillary improves to $\sim 0.02 \mathrm{~s}$ in water flow at $0.5 \mathrm{~m} / \mathrm{s}$.

Special care must be taken to ensure measurement accuracy because sensors respond to strain and humidity as well as temperature. Control or elimination of strain is the primary concern for temperature measurements and can be problematic since sensors require physical supports. Vertical sensor orientation, in which a small weight is suspended from the end to maintain constant tension, was tested and functioned well. But spanning the entire tank plane in this way with a single sensor would be difficult. Alternatively, sensors could be placed in capillaries to decouple them from support loads and reduce vibration, but at the expense of slowing response time by increasing the effective size and mass of the sensor.

Quantifying measurement accuracy of whole-field techniques such as LIF and PIV can be challenging, and the DTS has comparable issues. In this study, calibration was achieved by linking baseline readings to a single thermocouple, which was adequate because the DTS was baselined in a closed, stagnant and nearly isothermal system. But it may be difficult to obtain such temperature uniformity in other applications. In general, ensuring high accuracy along the entire length of a very long DTS is likely to require special care. And rigorous control of strain after baselining can be problematic for a DTS suspended in a flow field. Both these issues are exacerbated as flow turbulence and sensor size grow. There is a need to improve the optical fibers themselves for temperature sensing applications by reducing or eliminating strain and humidity sensitivity. Such work could investigate new coatings and clad/ core dimensions to improve repeatability and reduce drift associated with handling.
Acknowledgments The authors would like to thank Tyler Gorney at Luna Inc. for consultation and invaluable technical exchanges. The submitted manuscript has been created by UChicago Argonne, LLC, Operator of Argonne National Laboratory ("Argonne"). Argonne, a U.S. Department of Energy Office of Science laboratory, is operated under Contract No. DE-AC02-06CH11357. This work was supported by the U.S. Department of Energy, Office of Nuclear Energy.

Open Access This article is distributed under the terms of the Creative Commons Attribution License which permits any use, distribution, and reproduction in any medium, provided the original author(s) and the source are credited.

\section{References}

Abram C, Fond B, Heyes AL, Beyrau F (2013) High-speed planar thermometry and velocimetry using thermographic phosphor particles. Appl Phys B 111:155-160

Ahmad J, Purbolaksono J, Beng LC (2010) Thermal fatigue and corrosion fatigue in heat recovery area wall side tubes. Eng Fail Anal 17:334-343

Allen MG (1998) Diode laser absorption sensors for gas-dynamic combustion flows. Meas Sci Technol 9:545-562

Bao X, Chen L (2012) Recent progress in distributed fiber optic sensors. Sensors 12:8601-8639. doi:10.3390/s120708601

Berruti G, Consales M, Giordano M, Sansone L, Petagna P, Buontempo S, Breglio G, Cusano A (2013) Radiation hard humidity sensors based on polyimide-coated fiber Bragg gratings. In: Proceedings of SPIE, vol 8794, 5th European workshop on optical fibre sensors. doi: $10.1117 / 12.2026023$

Bhargava P, Chuang CK, Chen K, Zehnder A (2006) Moisture diffusion properties of HFPE-II-52 polyimide. J Appl Polymer Sci 102(4):3471-3479

Bhatia V (2001) Properties and applications of fiber gratings. Proc Int Conf Fiber Optics Photonics SPIE 4417:154160

Blondet E, Faidy C (2002) High cycle thermal fatigue in French PWR. Proceedings ICONE10 10th Int Conf Nuc Eng Arlington, VA April $14-18$ paper 22762

Boguszko M, Elliott G (2005) On the use of filtered Rayleigh scattering for measurements in compressible flows and thermal fields. Exp Fluids 38:33-49

Bohlin A, Kliewer CJ (2014) Diagnostic imaging in flames with instantaneous planar coherent Raman spectroscopy. J Phys Chem Lett 5:1243-1248

Bryant RA, Donbar JM, Driscoll JF (2000) Acetone laser induced fluorescence for low pressure/low temperature flow visualization. Exp Fluids 28:471-476

Buchhold R, Nakladal A, Gerlach G, Sahre K, Eichhorn KJ, Herold M, Gauglitz G (1998) Influence of moisture-uptake on mechanical properties of polymers used in microelectronics. Mat Res Soc Symp Proc 511:359-364

Cai T, Jia H, Wang G, Chen W, Gao X (2009) A sensor for measurements of temperature and water concentration using a single tunable diode laser near $1.4 \mu \mathrm{m}$. Sens Actuators A 152:5-12

Cehlin M, Moshfegh B, Sandberg M (2002) Measurements of air temperatures close to a low-velocity diffuser in displacement ventilation using an infrared camera. Energy Build 34:687-698

Cundy M, Trunk P, Dreizler A, Sick V (2011) Gas-phase toluene LIF temperature imaging near surfaces at $10 \mathrm{kHz}$. Exp Fluids 51:1169-1176

Dabiri D (2009) Digital particle image thermometry/velocimetry: a review. Exp Fluids 46:191-241

Dahikar SK, Sathe MJ, Joshi JB (2010) Investigation of flow and temperature patterns in direct contact condensation using PIV, PLIF and CFD. Chem Eng Sci 65:4606-4620 
Ding F, Wang L, Fang N, Huang Z (2011) Experimental study on humidity sensing using a FBG sensor with polyimide coating. SPIE-Int Soc. Opt Eng. 7990 Optical Sensors Biophotonics II 79900C

Fissolo A, Amiable S, Ancelet O, Mermaz F, Stelmaszyk JM, Constantinescu A, Robertson C, Vincent L, Maillot V, Bouchet F (2009) Crack initiation under thermal fatigue: an overview of CEA experience. Part I: thermal fatigue appears to be more damaging than uniaxial isothermal fatigue. Int J Fatigue 31:587-600

Froggatt M, Moore J (1998) Distributed measurement of static strain in an optical fiber with multiple Bragg gratings at nominally equal wavelengths. Appl Optics 37(10):1741-1746

Froggatt ME, Gifford DK, Kreger S, Wolfe M, Soller BJ (2006) Characterization of polarization-maintaining fiber using high-sensitivity optical-frequency-domain reflectometry. J Lightwave Tech 24(11):4149-4154

Fromme M, Christiansen W, Kjaer SV, Hill W (2011) Distributed temperature monitoring of long distance submarine cables. Proc SPIE Int Soc Opt Eng 7753(77532U):1-4

Galpin J, Simoneau JP (2011) Large eddy simulation of a thermal mixing tee in order to assess the thermal fatigue. Int $\mathrm{J}$ Heat Fluid Flow 32:539-545

Gifford DK, Kreger ST, Sang AK, Froggatt ME, Duncan RG, Wolfe MS, Soller BJ (2007) Swept-wavelength interferometric interrogation of fiber Rayleigh scatter for distributed sensing applications. Proc SPIE 6770 Fiber Optic Sens Appl V 67700F

Gordge DN, Page RH (1993) Infrared imagery of an air/CO $\mathrm{CO}_{2}$ axisymmetric jet. Exp Fluids 14:409-415

Hannink MHC, Blom FJ (2011) Numerical methods for the prediction of thermal fatigue due to turbulent mixing. Nucl Eng Design 241:681-687

Hu DC, Chen HC (1992) Humidity effect on polyimide film adhesion. J Mat Sci 27:5262-5268

Kreger ST, Gifford DK, Froggatt ME, Soller BJ, Wolfe MS (2006) High resolution distributed strain or temperature measurements in single- and multi-mode fiber using swept-wavelength interferometry. Optic Fiber Sens OSA/OFS 2006, p 4

Kulatilaka WD, Stauffer HU, Gord JR, Roy S (2011) One-dimensional single-shot thermometry in flames using femtosecondCARS line imaging. Opt Lett 36(21):4182-4184

Lee SB (1995) Thermal fatigue failure and design modification of a steam exhaust silencer. Eng Fail Anal 2(4):247-255

Liu X, Jeffries JB, Hanson RK (2007) Measurement of nonuniform temperature distributions using line-of-sight absorption spectroscopy. AIAA J 45(2):411-419

Lomperski S, Gerardi C (2014) Assessment of distributed fiber optic sensors for flow field temperature mapping. In: Proc. ASME 2014 4th Joint US-European Fluids Engineering Division Summer Meeting, 3-7 August, Chicago, IL, paper FEDSM2014-22156
Lomperski S, Gerardi C, Pointer DW (2013) Distributed fiber optic temperature sensing for CFD code validation. In: Proceedings of 15th International Topical Meeting on Nuclear Reactor Thermal Hydraulics (NURETH-15) Pisa, Italy, 12-17 May, paper 374

Narayanan V, Page RH, Seyed-Yagoobi J (2003) Visualization of air flow using infrared thermography. Exp Fluids 34:275-284

Neto LPC, Gameiro Silva MC, Costa JJ (2006) On the use of infrared thermography in studies with air curtain devices. Energy Build 38:1194-1199

Palmieri L, Schenato L (2013) Distributed optical fiber sensing based on Rayleigh scattering. Open Optics J 7(Suppl-1, M7):104-127

Rajeev P, Kodikara J, Chiu WK, Kuen T (2013) Distributed optical fibre sensors and their applications in pipeline monitoring. Key Eng Mater 558:424-434

Salonen J, Auerkari P, Lehtinen O, Pihkakoski M (2007) Experience on in-service damage in power plant components. Eng Fail Anal 14:970-977

Shafii MB, Lum CL, Koochesfahni MM (2010) In situ LIF temperature measurements in aqueous ammonium chloride solution during uni-directional solidification. Exp Fluids 48:651-662

Someya S, Yoshida S, Li Y, Okamoto K (2009) Combined measurement of velocity and temperature distributions in oil based on the luminescent lifetimes of seeded particles. Meas Sci Techol 20:025403. doi:10.1088/0957-0233/20/2/025403

Sou IM, Allen JS, Layman CN, Ray C (2011) A synchronized particle image velocimetry and infrared thermography technique applied to an acoustic streaming flow. Exp Fluids 51:1201-1208

Uttam D, Culshaw B (1985) Precision time domain reflectometry in optical fiber systems using a frequency modulated continuous wave ranging technique. J Lightwave Tech 5:971-977

Volino RJ, Smith GB (1999) Use of simultaneous IR temperature measurements and DPIV to investigate thermal plumes in a thick layer cooled from above. Exp Fluids 27:70-78

Williams G, Brown G, Hawthorne W, Hartog A, Waite P (2000) Distributed temperature sensing (DTS) to characterize the performance of producing oil wells. Proc SPIE Int Soc Opt Eng 4202:39-54

Yetisir M (2009) Thermal fatigue in CANDU stations. In: Proceedings of 17th Int Con Nuc Eng ICONE17, Brussels, Belgium, 12-16 July, paper 75603

Yúksel K, Mégret P, Wuilpart M (2011) A quasi-distributed temperature sensor interrogated by optical frequencydomain reflectometer. Meas Sci Technol 22:115204. doi:10.1088/0957-0233/22/11/115204

Zetterberg J, Li Z, Afzelius M, Aldén M (2008) Two-dimensional temperature measurements in flames using filtered Rayleigh scattering at $254 \mathrm{~nm}$. Appl Spectrosc 62(7):778-783 